\title{
THE SPECTRUM OF PERIODIC POINT PERTURBATIONS AND THE KREIN RESOLVENT FORMULA
}

\author{
J. BRÜNING and V. A. GEYLER
}

\begin{abstract}
We study periodic point perturbations, $H$, of a periodic elliptic operator $H^{0}$ on a connected complete non-compact Riemannian manifold $X$, endowed with an isometric, effective, properly discontinuous, and co-compact action of a discrete group $\Gamma$. Under some conditions on $H^{0}$, we prove that the gaps of the spectrum are labelled in a natural way by elements of the $K_{0}$-group of a certain $C^{*}$-algebra. In particular, if the group $\Gamma$ has the Kadison property then the spectrum has band structure. The Krein resolvent formula plays a crucial role in proving the main results.
\end{abstract}

\section{Introduction}

The spectral analysis of periodic Schrödinger operators poses an interesting problem in physics and mathematics. Among these operators, those with point potential play an important role in view of the fact that the corresponding spectral problem is explicitly solvable [1], [2]. Thus, almost all textbooks on condensed matter physics refer to the well-known Kronig - Penney model [3]. This model was generalized to two and three dimensions in [4] - [6] (see also [2] for details and further references).

On the other hand, investigations of periodic elliptic operators (including Schrödinger operators) on complete Riemannian manifolds have begun in the last decade. Using K-theory for $C^{*}$-algebras, J.Brüning and T.Sunada have studied the band structure of the spectrum for such the operators $[7],[8],[9]$. The results of the cited papers are based on the analysis of heat kernels. For the case of point perturbations, the heat kernel of the perturbed operator has a complicated form; therefore, we study the resolvent here. The famous Krein resolvent formula [10] provides an adequate tool for obtaining and analyzing the resolvent of a Schrödinger operator perturbed by a point potential. As a result we show in this paper that under certain natural conditions the Krein formula works for the case of point perturbations of elliptic operators on a manifold, too. With the help of this formula we prove that the gaps of a periodic point perturbation of such an operator are labelled by the elements of the $K_{0}$-group of an appropriate $C^{*}$-algebra. These results may be generalized to the case of gauge-periodic point perturbations of larger classes of elliptic operators [11].

In conclusion, we note that the spectral analysis of periodic Schrödinger operators on manifolds of non-zero curvature is necessary in understanding many physical phenomena 
like quantum chaos $([12],[13])$ and charge transport in non-planar systems [14].

\section{Preliminaries}

Throughout the paper $X$ denotes a connected complete non-compact Riemannian $C^{\infty}$ manifold of dimension $n$; $\Gamma$ denotes a discrete group which acts on $X$ isometrically, effectively, and properly discontinuously with compact quotient $\Gamma \backslash X$. We shall denote by $d(x, y)$ the Riemannian distance on $X$ and by $d x$ the mesure on $X$ associated with the Riemannian metric; of course, $d x$ is a $\Gamma$-invariant measure. It is known (see, e.g., [15]) that there exists a set $F$ (called the Brillouin zone for $\Gamma$ ) with the properties:

1) $F$ is an open and connected set with a negligible boundary;

2) $\gamma F \cap F=\emptyset$ if $\gamma \neq e$;

3) $\bar{F}$ is compact, the system $(\gamma \bar{F})_{\gamma \in \Gamma}$ is locally finite, and

$$
\bigcup_{\gamma \in \Gamma} \gamma \bar{F}=X
$$

By $L$ we shall denote the standard representation $\Gamma$ in $L^{2}(X)$; for $\gamma \in \Gamma, L_{\gamma}$ is a unitary operator acting by the rule $L_{\gamma} f(x)=f\left(\gamma^{-1} x\right)$.

Let $\tau_{0}: C_{0}^{\infty}(X) \rightarrow C_{0}^{\infty}(X)$ be a $\Gamma$-invariant formally self-adjoint elliptic operator of order $m, m>n / 2$. The closure, $H^{0}$, of $\tau_{0}$ in the Hilbert space $\mathcal{H}=L^{2}(X)$ is a self-adjoint operator with domain $\mathcal{D}\left(H^{0}\right):=W_{2}^{m}(X)[8],[16]$. Note that

$$
\mathcal{D}\left(H^{0}\right) \subset C(X)
$$

in view of the Sobolev embedding theorem. By spec $A$ we denote, as usual, the spectrum of a closed operator $A$ and we put $\rho(A):=\mathbf{C} \backslash \operatorname{spec} A$. For $\zeta \in \rho\left(H^{0}\right), R^{0}(\zeta):=\left(H^{0}-\zeta\right)^{-1}$ denotes the resolvent of $H^{0}$. It follows from (1) that $R^{0}(\zeta)$ is a bi-Carleman operator for every $\zeta \in \rho\left(H^{0}\right)$ [17]. Recall that a bounded operator $A$ in $L^{2}(X)$ is called a bi-Carleman operator if there is a measurable function $K_{A}: X \times X \rightarrow \mathbf{C}$ (the integral kernel of $A$ ) such that for any $f \in L^{2}(X)$

$$
A f(x)=\int_{X} K_{A}(x, y) f(y) d y \text { for a.e. } x
$$

and

$$
\int_{X}\left|K_{A}(x, y)\right|^{2} d y<+\infty \quad \text { for a.e. } x, \quad \int_{X}\left|K_{A}(x, y)\right|^{2} d x<+\infty \text { for a.e. } y
$$

(see, e.g., [18] for details). We denote by $G^{0}(x, y ; \zeta)$ the integral kernel of $R^{0}(\zeta)$ and by $G^{1}\left(x, y ; \zeta_{1}, \zeta_{2}\right)$ the iterated kernel

$$
G^{1}\left(x, y ; \zeta_{1}, \zeta_{2}\right)=\int_{X} G^{0}\left(x, u ; \zeta_{1}\right) G^{0}\left(u, y ; \zeta_{2}\right) d u .
$$

In what follows we shall suppose that the principal symbol $a_{m}$ of $\tau_{0}$ satisfies the AgmonAgranovich-Vishik condition: 
(AAV) There exists a constant $C>0$ such that $\left|a_{m}(\nu)+\lambda\right| \geq C$ for all $\lambda>0$ and all $\nu \in T^{*} X$ with $|\nu|=1$.

The following result is proved in [16] (see Lemmas 4.5, 4.6, and Theorem 4.7); it allows to employ the Krein resolvent formula to point perturbations of $H^{0}$ :

Theorem A. (1) There exists $\tilde{E} \in \mathbf{R}, \tilde{E}<0$, such that for $E<\tilde{E}$ the kernel $G^{0}(x, y ; E)$ is $a C^{\infty}$-function outside the diagonal $x=y$.

(2) The operator $H^{0}$ is semibounded from below. Moreover, for every $t>0$ there exist constants $E_{0}(t)<0$ and $k_{0}(t)>0$ such that for any $x, y \in X, x \neq y$, and for $E<E_{0}$

$$
\left|G^{0}(x, y ; E)\right| \leq k_{0} d(x, y)^{m-n} \exp (-t d(x, y))
$$

if $m<n$, and

$$
\left|G^{0}(x, y ; E)\right| \leq k_{0}\left(1+d(x, y)^{m-n}|\log (d(x, y))|\right) \exp (-t d(x, y))
$$

otherwise.

To prove Lemma 1 below we need the statement [16]:

Lemma B. Let $B(x, r)=\{y \in X: d(x, y)<r\}$. There exists a constant $C_{X}$ such that $\operatorname{Vol}(B(x, r)) \leq \exp \left(C_{X} r\right)$ for all $x \in X$ and $r>0$.

Lemma 1. The following assertions are valid.

(1) There is a constant $\widehat{E}<0$ such that for any $\zeta_{1}, \zeta_{2} \in \rho\left(H^{0}\right)$ the function $G^{1}\left(x, y ; \zeta_{1}, \zeta_{2}\right)$ is at least separately continuous on $X \times X$ if $\zeta_{2}<\widehat{E}$ or $\zeta_{1}<\widehat{E}$.

(2) For fixed $\zeta \in \rho\left(H^{0}\right)$ the function $G^{0}(x, y ; \zeta)$ is at least separately continuous on $X \times X$ outside the diagonal $x=y$.

(3) For every $\varepsilon>0$ and $t>0$ there exist constants $E_{1}(t, \varepsilon)<0$ and $k_{1}(t, \varepsilon)>0$ such that for $d(x, y) \geq \varepsilon$

$$
\left|G^{0}(x, y ; E)\right| \leq k_{1} \exp (-t d(x, y))
$$

whenever $E<E_{1}$, and

$$
\left|G^{1}\left(x, y ; E^{\prime}, E^{\prime \prime}\right)\right| \leq k_{1} \exp (-t d(x, y))
$$

whenever $E^{\prime}, E^{\prime \prime}<E_{1}$.

(4) Let $K$ be a compact subset of $X$ and $x_{0}$ be a point of $X$. Then for every $\varepsilon>0$ and $t>0$ there exist constants $E_{2}(t, \varepsilon)<0$ and $k_{2}\left(t, \varepsilon, K, x_{0}\right)>0$ such that for $E<E_{2}$

$$
\sup \left\{\left|G^{0}(x, y ; E)\right|: y \in K\right\} \leq k_{2} \exp \left(-t d\left(x, x_{0}\right)\right)
$$

whenever $d(x, K) \geq \varepsilon$.

(5) Let $K$ be a compact subset of $X$ and $x_{0}$ be a point of $X$. Then for every $t>0$ there exist constants $E_{3}(t)<0$, and $k_{3}\left(t, K, x_{0}\right)>0$ such that for $E<E_{3}$

$$
\left[\int_{K}\left|G^{0}(x, y ; E)\right|^{2} d y\right]^{1 / 2} \leq k_{3} \exp \left(-t d\left(x, x_{0}\right)\right)
$$


whenever $d(x, K) \geq \varepsilon$.

Proof. Using Theorem A, Lemma $\mathrm{B}$, and the fact that $G^{0}(x, y ; \zeta)$ is a bi-Carleman kernel for every $\zeta \in \rho\left(H^{0}\right)$, it is not hard to prove that there is a constant $E^{1}<0$ such that for any $\zeta_{1}, \zeta_{2} \in \rho\left(H^{0}\right)$ we have the following: if $\zeta_{2}<E^{1}$, then the function $G^{1}\left(x, y ; \zeta_{1}, \zeta_{2}\right)$ is continuous with respect to $y$ for fixed $x$, and if $\zeta_{1}<E^{1}$, then this function is continuous with respect to $x$ for fixed $y$. To complete the proof of the assertion (1) it is sufficient to apply the identity

$$
\overline{G^{1}\left(x, y ; \zeta_{1}, \zeta_{2}\right)}=G^{1}\left(y, x ; \bar{\zeta}_{1}, \bar{\zeta}_{2}\right) .
$$

The assertion (2) is a consequence of (1) and the Hilbert resolvent identity. The first inequality in (3) immediately follows from Theorem A; the second one is a simple corollary of the first inequality and Lemma B. The proof of the statements (4) and (5) is trivial in virtue of the first inequality in (3).

In the remainder of this section, we present some necessary facts from M.G.Krein's theory of self-adjoint extensions (see [10] for more details).

Let $H^{0}$ be a self-adjoint operator in a Hilbert space $\mathcal{H}, S$ a symmetric operator which is a restriction of $H^{0}$, and let $\mathcal{N}_{\zeta}=\operatorname{Ker}\left(S^{*}-\zeta\right)$, where $\zeta \in \rho\left(H^{0}\right)$, be the deficiency subspace of $\bar{S}$. Fix a Hilbert space $\mathcal{G}$ with $\operatorname{dim} \mathcal{G}=\operatorname{dim} \mathcal{N}_{\zeta}$. A mapping $\zeta \mapsto B(\zeta)$ from $\rho\left(H^{0}\right)$ to the space $\mathcal{L}(\mathcal{G}, \mathcal{H})$ of all bounded operators from $\mathcal{G}$ to $\mathcal{H}$ is called a Krein $\Gamma$-field of the pair $\left(H^{0}, S\right)$ if the following conditions are satisfied:

$(\Gamma 1) B(\zeta)$ is a linear topological isomorphism of $\mathcal{G}$ onto $\mathcal{N}_{\zeta}$;

(Г2) with

$$
U(\zeta, z):=\left(H^{0}-\zeta\right)\left(H^{0}-z\right)^{-1}
$$

we have

$$
B(z)=U(\zeta, z) B(\zeta)
$$

If we choose an arbitrary linear topological isomorphism $B\left(z_{0}\right): \mathcal{G} \rightarrow \mathcal{N}_{\zeta_{0}}$, we can uniquely determine a $\Gamma$-field $B$ by $B(z)=U\left(z_{0}, z\right) B\left(z_{0}\right)$. A mapping $Q: \rho\left(H^{0}\right) \rightarrow \mathcal{L}(\mathcal{G}, \mathcal{G})$ is said to be a Krein $\mathcal{Q}$-function if

$$
Q(\zeta)-Q(z)^{*}=(\zeta-\bar{z}) B(z)^{*} B(\zeta)
$$

for each $z, \zeta \in \rho\left(H^{0}\right)$. It follows from Eqs. (3)-(5) that $Q(z)$ is a holomorphic operatorvalued function of $\zeta$. This function is uniquely determined by the property (5) up to a self-adjoint summand $C \in \mathcal{L}(\mathcal{G}, \mathcal{G})$. If $C$ in $\mathcal{L}(\mathcal{G}, \mathcal{G})$ is given, we can put

$$
Q(z)=C-i y_{0} B\left(z_{0}\right)^{*} B\left(z_{0}\right)+\left(z-\bar{z}_{0}\right) B\left(z_{0}\right)^{*} B(z),
$$

where $z_{0}$ is a fixed element of $\rho\left(H^{0}\right)$ and $y_{0}=\operatorname{Im} z_{0}$. Recall that a self-adjoint extension $H$ of $S$ is called disjoint from $H^{0}$ if $\mathcal{D}(H) \cap \mathcal{D}\left(H^{0}\right)=\mathcal{D}(S)$. The following theorem is the main result of the Krein theory of self-adjoint extensions (see [10] for the proof).

Theorem C. Given an arbitrary self-adjoint (not necessarily bounded) operator $A$ in $\mathcal{G}$, the formula

$$
R_{A}(z)=R^{0}(z)-B(z)[Q(z)+A]^{-1} B(\bar{z})^{*}
$$


determines the resolvent of a self-adjoint extension, $H_{A}$, of $S$ that is disjoint from $H^{0}$. Moreover, the correspondence $A \mapsto H_{A}$ estabilishes a bijection between the set of all selfadjoint extensions of $S$ disjoint from $H^{0}$ and the set of all self-adjoint operators in $\mathcal{G}$.

Below we need the following property of the operators $U(\zeta, z)$ :

Proposition D. The mapping $U(\zeta, z)$ is a linear topological isomorphism of the space $\mathcal{N}_{\zeta}$ onto $\mathcal{N}_{z}$ given by $U(\zeta, z)=I+(z-\zeta) R^{0}(z)$.

\section{Periodic point perturbations of $H^{0}$}

Let us fix a fundamental domain $F$ and some finite subset $\mathrm{K}$ of $F$, and let $\Lambda$ be the $\Gamma$-orbit of $\mathrm{K}: \Lambda=\bigcup_{\gamma \in \Gamma} \gamma \mathrm{K}$. The set $\Lambda$ may be viewed as the analog of a crystal in Euclidean space. It follows from the properties of the domain $F$ that each point $\lambda \in \Lambda$ has a unique representation of the form $\lambda=\gamma \kappa$ where $\gamma \in \Gamma, \kappa \in \mathrm{K}$. Now we define a point perturbation of $H^{0}$ supported by $\Lambda$ : Formally, this is a self-adjoint operator $H$ of the form

$$
H=H^{0}+\sum_{\substack{\gamma \in \Gamma \\ \kappa \in \mathrm{K}}} \varepsilon_{\kappa} \delta_{\gamma \kappa}(x),
$$

where $\delta_{y}$ is the Dirac $\delta$-function supported at $y \in X$ and $\varepsilon_{\kappa}$ are "coupling constants". To assign an operator meaning to the formal expression (8), we use the so-called "restrictionextension procedure" [1], [2]. Thus, we consider the set

$$
\mathcal{D}(S)=\left\{f \in \mathcal{D}\left(H^{0}\right): f(\lambda)=0, \quad \lambda \in \Lambda\right\}
$$

which is well defined since $\mathcal{D}\left(H^{0}\right) \subset C(X)$. Let $S$ be the restriction of $H^{0}$ to $\mathcal{D}(S)$; evidently, $S$ is a symmetric operator in $\mathcal{H}$. A self-adjoint extension $H$ of $S$ disjoint from $H^{0}$ is then said to be a point perturbation of $H^{0}$ supported by $\Lambda$.

Fix a point perturbation $H$ of $H^{0}$. Using the Krein resolvent formula (7) we construct an explicit form of the resolvent $R(\zeta)$ of $H$ for which we need some results from [19]. These results are obtained for the case $\mathcal{H}=L^{2}(\Omega), \Omega$ a domain in $\mathbf{R}^{n}$, but it is easy to check that they are also valid for the case $\mathcal{H}=L^{2}(M)$ where $M$ is an arbitrary locally compact space together with a Radon measure.

In what follows we shall denote by $I_{0}$ the semi-axis $(-\infty, \tilde{E})$, with $\tilde{E}$ the constant from Theorem A. Let $\zeta \in I_{0}$; for every $a \in X$ we denote by $g_{a}(\zeta)$ the function $X \ni$ $x \mapsto G^{0}(x, a ; \zeta)$; if $z \in \rho\left(H^{0}\right)$ is arbitrary, then we put $g_{a}(z)=U(\zeta, z) g_{a}(\zeta)$. In view of Proposition D, this definition of $g_{a}(z)$ does not depend on the choice of $\zeta$ in $I_{0}$.

Lemma 2. For some $z \in I_{0}$ the matrix $\left(\left\langle g_{\lambda}(z) \mid g_{\mu}(z)\right\rangle\right)_{\lambda, \mu \in \Lambda}$ determines a bounded operator in the standard basis of the space $l^{2}(\Lambda)$.

Proof. It follows from Lemma $B$ that there are constants $c_{\Lambda}>0$ and $\tilde{c}_{\Lambda}>0$ such that for all $\lambda \in \Lambda$ and $r \in \mathbf{R}_{+}$we have

$$
\#\{\mu \in \Lambda: d(\lambda, \mu) \leq r\} \leq c_{\Lambda} \exp \left(\tilde{c}_{\Lambda} r\right)
$$

where $\# Y$ is the number of elements in a finite set $Y$. Denote for simplicity $\left\langle g_{\lambda}(z) \mid g_{\mu}(z)\right\rangle$ by $W(\lambda, \mu)$. According to Schur's test $[20]$, the operator $W$ with the matrix $W(\lambda, \mu)$ is bounded 
on the space $l^{2}(\Lambda)$ if for some $c^{\prime}>0$

$$
\sup _{\mu \in \Lambda} \sum_{\lambda \in \Lambda}|W(\lambda, \mu)| \leq c^{\prime} \quad \text { and } \quad \sup _{\lambda \in \Lambda} \sum_{\mu \in \Lambda}|W(\lambda, \mu)| \leq c^{\prime},
$$

and in this case we have $\|W\| \leq c^{\prime}$. To find such a constant $c^{\prime}$ it is sufficient to use Lemma $1(3)$ and the following assertion which is proved in [21]:

Lemma E. Let $\varphi: \Lambda \rightarrow \mathbf{C}$ be a function such that for some $\mu \in \Lambda$

$$
|\varphi(\lambda)| \leq c \exp \left(-(1+\delta) \tilde{c}_{\Lambda} d(\lambda, \mu)\right)
$$

where $c$ and $\delta$ are positive constants. Then

$$
\sum_{\lambda \in \Lambda}|\varphi(\lambda)| \leq c \tilde{c}_{\Lambda} \delta^{-1}
$$

For each $\kappa \in \mathrm{K}$ we choose a function $\varphi_{\kappa} \in C_{0}^{\infty}(X)$ such that $\varphi_{\kappa}(\kappa)=1, \operatorname{supp} \varphi_{\kappa} \subset$ $F$, and $\operatorname{supp} \varphi_{\kappa} \cap \operatorname{supp} \varphi_{\kappa^{\prime}}=\emptyset$ if $\kappa \neq \kappa^{\prime}$. For every $\lambda \in \Lambda$ we put $\varphi_{\lambda}=L_{\gamma} \varphi_{\kappa}$, if $\lambda=\gamma \kappa$. It is readily seen that the family $\left\{\varphi_{\lambda}: \lambda \in \Lambda\right\}$ lies in $\mathcal{D}\left(H^{0}\right)$ and possesses the properties:

(1) $\varphi_{\lambda}(\lambda)=1(\lambda \in \Lambda)$;

(2) $\operatorname{supp} \varphi_{\lambda} \cap \operatorname{supp} \varphi_{\lambda^{\prime}}=\emptyset$ if $\lambda \neq \lambda^{\prime}$;

(3) $\sup \left\{\left\|H^{0} \varphi_{\lambda}\right\|+\left\|\varphi_{\lambda}\right\|: \lambda \in \Lambda\right\}<\infty$.

Taking into account Lemma 2, we can apply Theorem 3 and Proposition 3 from [19] and get the following result:

Proposition 1. For every $z \in \rho\left(H^{0}\right)$ the family $\left\{g_{\lambda}(z): \lambda \in \Lambda\right\}$ is a Riesz basis in $\mathcal{N}_{z}$. This means that for each family $\left(\xi_{\lambda}\right)_{\lambda \in \Lambda}$ from $l^{2}(\Lambda)$ the family $\left(\xi_{\lambda} g_{\lambda}(z)\right)_{\lambda \in \Lambda}$ is summable in $\mathcal{H}$ and the mapping

$$
B(z): l^{2}(\Lambda) \ni\left(\xi_{\lambda}\right) \mapsto \sum_{\lambda \in \Lambda} \xi_{\lambda} g_{\lambda}(z) \in \mathcal{H}
$$

is a linear topological isomorphism from $l^{2}(\Lambda)$ onto $\mathcal{N}_{z}$.

Now we put $\mathcal{G}:=l^{2}(\Lambda)$; using (11) and Proposition D it is easily shown that $B(z)$ is a Krein $\Gamma$-field.

Our next purpose is to construct the Krein $\mathcal{Q}$-function for the pair $\left(H^{0}, S\right)$. Fix a point $z_{0} \in \mathbf{R}$ such that $z_{0}<\widehat{E}$ (cf. Lemma $1(1)$ ), then for all $z \in \rho\left(H^{0}\right)$ and all $a \in X$ the expression $G^{0}(a, a ; z)-G^{0}\left(a, a ; z_{0}\right)$ is well-defined. Indeed, from the Hilbert resolvent identity we get

$$
G^{0}(a, a ; z)-G^{0}\left(a, a ; z_{0}\right)=\left(z-z_{0}\right) G^{1}\left(a, a ; z, z_{0}\right) .
$$

Now, using Theorem 4 and Proposition 4 from [19] we can determine the Krein $\mathcal{Q}$-function by the infinite matrix $\left(Q_{\lambda \mu}(z)\right)_{\lambda, \mu \in \Lambda}$ if

$$
Q_{\lambda \mu}(z):= \begin{cases}G^{0}(\lambda, \mu ; z), & \text { if } \lambda \neq \mu \\ G^{0}(\lambda, \lambda ; z)-G^{0}\left(\lambda, \lambda ; z_{0}\right), & \text { if } \lambda=\mu .\end{cases}
$$

The results thus obtained are summarized in the following theorem. 
Theorem 1. Let $H_{A}$ be the point perturbation of $H^{0}$ determined by a self-adjoint operator $A$ in the space $l^{2}(\Lambda)$. Then for every $\zeta \in \rho\left(H^{0}\right) \cap \rho\left(H_{A}\right)$ and $f \in L^{2}(X)$ we have

$$
R_{A}(\zeta) f=R^{0}(\zeta) f-\sum_{\lambda \in \Lambda}\left(\sum_{\mu \in \Lambda}[Q(\zeta)+A]^{-1}(\lambda, \mu)\left\langle g_{\mu} \mid f\right\rangle\right) g_{\lambda}(\zeta)
$$

We are interested in $\Gamma$-periodic point perturbations of $H^{0}$ only. Proposition 2 below provides a necessary and sufficient condition for $H_{A}$ to be a $\Gamma$-invariant operator. Before stating this proposition we note that there is a natural unitary representation $\tilde{L}$ of the group $\Gamma$ in $l^{2}(\Lambda): \tilde{L}_{\gamma} \varphi(\lambda)=\varphi\left(\gamma^{-1} \lambda\right), \varphi \in l^{2}(\Lambda)$. It is clear that for each $z \in \rho\left(H^{0}\right)$ the operator $Q(z)$ is $\tilde{L}$-invariant, that is, its matrix satisfies the condition $Q_{\lambda+\gamma, \mu+\gamma}(z)=Q_{\lambda \mu}(z)$ for all $\gamma \in \Gamma, \lambda, \mu \in \Lambda$. In particular, the diagonal elements $Q_{\lambda \lambda}(z)$ depend only on $\mathrm{K}$ since we have $\lambda=\gamma \kappa$ for some $\gamma \in \Gamma$ and $\kappa \in \mathrm{K}$.

Proposition 2. The operator $H_{A}$ is $\Gamma$-periodic if and only if the operator $A$ is invariant with respect to $\tilde{L}$.

We omit the easy proof of this proposition.

From now on, we consider only $\Gamma$-periodic point perturbations, $H_{A}$, of $H^{0}$. From the point of view of physical applications, the most important operators $H_{A}$ are those where $A$ has a diagonal matrix with respect to the standard basis of $l^{2}(\Lambda)$ [4], [22]; only these operators appear as limits of Hamiltonians with short-range potentials [4], [23]. On the other hand, even in the case of a bounded $\tilde{L}$-invariant operator $A$ with a non-diagonal matrix, the spectrum of the periodic point perturbation $H_{A}$ for the Laplacian $H^{0}=-\Delta$ may contain a singular component which is a Cantor set [24]. For this reason, we restrict ourselves to the case when the following conditions are fulfilled:

(D) The operator $A$ has a diagonal matrix $A_{\lambda \mu}=\alpha_{\lambda \mu} \delta_{\lambda \mu}, \quad \lambda, \mu \in \Lambda$;

(Q) $\lim _{E \rightarrow \infty}\left|Q_{\kappa \kappa}(E)\right|=\infty$ for all $\kappa \in \mathrm{K}$.

Yu. G. Shondin has observed (for the case of finite point perturbations) that the conditions (D) and (Q) eliminate some pathological properties of $H_{A}$ [25]. Namely, under these conditions the operator $H_{A}$ is "form-local" in the following sense: for any $\varphi$ and $\psi$ from the form-domain $Q\left(H_{A}\right)$ of $H_{A}$ the relation supp $\varphi \cap \operatorname{supp} \psi=\emptyset$ implies $\left\langle\varphi \mid H_{A} \psi\right\rangle=0$.

From now on, we shall suppose thath the condidtions (D) and (Q) are satisfied. The following theorem is the main result of this section.

Theorem 2. For every $t>0$ there are constants $E_{4}(t)<0$ and $k_{4}(t)>0$ such that for every $E \in \mathbf{R}, E<E_{4}$, the operator $Q(E)+A$ has a bounded inverse with matrix obeying the condition

$$
\left|[Q(z)+A]_{\lambda \mu}^{-1}\right| \leq k_{4} \exp (-t d(\lambda, \mu)) .
$$

Proof Let $t>0$ be given. Denote by $D(E)$ the operator in $l^{2}(\Lambda)$ with matrix $D_{\lambda \mu}(E)=$ $\left(Q_{\lambda \mu}(E)+A_{\lambda \mu}\right) \delta_{\lambda \mu}$, and set $S(E)=Q(E)+A-D(E)$. Let $C_{E}=\inf \left\{\left|D_{\lambda \lambda}(E)\right|: \lambda \in\right.$ $\Lambda$; ; according to the condition (Q), $C_{E} \rightarrow \infty$ as $E \rightarrow-\infty$. Let $s=\max \left(t, 2 \tilde{c}_{\Lambda}\right)$. By Lemma 1 there are $E_{4}<0$ and $c>0$ such that $\left|S_{\lambda \mu}(E)\right| \leq c \exp (-2 s d(\lambda, \mu))$, whenever 
$E<E_{4}$. We can suppose $\left|E_{4}\right|$ is so large that for $E<E_{4}$ we have $\tilde{c}_{\Lambda} c C_{E}^{-1} \leq 1 / 2$ and $\left\|D(E)^{-1}\right\|+\left\|D(E)^{-1} S(E)\right\|<1$. Then

$$
[Q(E)+A]^{-1}=\sum_{j \geq 0}\left(-D(E)^{-1} S(E)\right)^{j}
$$

We claim that for all $j \geq 0$

$$
\left|\left(D(E)^{-1} S(E)\right)_{\lambda \mu}^{j}\right| \leq\left(\tilde{c}_{\Lambda} c C_{E}^{-1}\right)^{j} \exp (-s d(\lambda, \mu))
$$

implying the theorem.

For $j=0$ we have nothing to prove. If the assertion holds for some $j \geq 0$, we estimate with Lemma $\mathrm{E}$

$$
\begin{aligned}
\left|\left(D(E)^{-1} S(E)\right)_{\lambda \mu}^{j+1}\right| & \leq C_{E}^{-1} \sum_{\kappa \in \Lambda}\left|S_{\lambda \kappa}(E)\left(D(E)^{-1} S(E)\right)_{\kappa \mu}^{j}\right| \\
& \leq c C_{E}^{-1}\left(\tilde{c}_{\Lambda} c C_{E}^{-1}\right)^{j} \sum_{\kappa \in \Lambda} \exp (-2 s d(\lambda, \kappa)) \exp (-s d(\kappa, \mu)) \\
& \leq c C_{E}^{-1}\left(\tilde{c}_{\Lambda} c C_{E}^{-1}\right)^{j} \exp (-s d(\lambda, \mu)) \sum_{\kappa \in \Lambda} \exp \left(-2 \tilde{c}_{\Lambda} d(\lambda, \kappa)\right) \\
& \leq\left(\tilde{c}_{\Lambda} c C_{E}^{-1}\right)^{j+1} \exp (-s d(\lambda, \mu)) .
\end{aligned}
$$

Corollary. The operator $H_{A}$ is semi-bounded from below.

3. Spectral structure of $H_{A}$.

In this section we denote by $\mathcal{K}$ the set of all compact operators in the space $\mathcal{F}=L^{2}(F)$. We put $C_{\text {red }}^{*}(\Gamma, \mathcal{K}):=C_{\text {red }}^{*}(\Gamma) \otimes \mathcal{K}$, where $C_{\text {red }}^{*}(\Gamma)$ is the reduced group $C^{*}$-algebra of $\Gamma[7],[8]$.

We shall identify $L^{2}(X)$ with the space $l^{2}(\Gamma, \mathcal{F})$ by means of the correspondence

$$
\begin{aligned}
& \Phi: L^{2}(X) \ni f \mapsto \Phi f \in l^{2}(\Gamma, \mathcal{F}), \\
& \Phi f(\gamma)=r_{F}\left(L_{\gamma} f\right)=r_{F}\left(f \circ \gamma^{-1}\right),
\end{aligned}
$$

with inverse

$$
\Phi^{-1} \phi=\sum_{\gamma \in \Gamma} L_{\gamma}^{*} e_{F}(\phi(\gamma))
$$

here $r_{F}$ is the restriction to $F$ and $e_{F}$ the extension by 0 . With $\tilde{R}$ the right regular representation of $\Gamma$ in $l^{2}(\Gamma)$ we set $R:=\tilde{R} \otimes I$, where $I$ is the identity.

Lemma 3. The mapping $\Phi$ is an interwining operator for the representations $L$ and $R$.

Proof. This follows by direct calculation.

This lemma implies that we can identify the space of all $\Gamma$-invariant operators in $\mathcal{L}(\mathcal{H})$ with the space $W^{*}(\Gamma, \mathcal{F})$ of all bounded $R$-invariant operators $B: l^{2}(\Gamma, \mathcal{F}) \rightarrow l^{2}(\Gamma, \mathcal{F})$, and that we can view $C_{\text {red }}^{*}(\Gamma, \mathcal{K})$ as a subalgebra of $W^{*}(\Gamma, \mathcal{F})$. For $B \in W^{*}(\Gamma, \mathcal{F})$ we define the Fourier coefficient $\widehat{B}(\gamma)$ at $\gamma \in \Gamma$ to be the bounded operator on $\mathcal{F}$ given by

$$
\widehat{B}(\gamma) v=\left(B \delta_{1}^{v}\right)(\gamma)
$$


where

$$
\delta_{1}^{v}(\gamma)= \begin{cases}v, & \text { if } \gamma=e, \\ 0 & \text { otherwise }\end{cases}
$$

Recall that the canonical trace, $\operatorname{Tr}_{\Gamma}$, on $C_{\text {red }}^{*}(\Gamma, \mathcal{K})$ is given by

$$
\operatorname{Tr}_{\Gamma} B=\operatorname{Tr} \hat{B}(e) .
$$

We need the following lemma [8]:

Lemma F. If $\widehat{B}(\gamma) \in \mathcal{K}$ for every $\gamma \in \Gamma$ and

$$
\sum_{\gamma \in \Gamma}\|\hat{B}(\gamma)\|<\infty
$$

then $B \in C_{\text {red }}^{*}(\Gamma, \mathcal{K})$.

Our main results are consequences of the following theorem.

Theorem 3. The resolvent, $R_{A}(\zeta)$, of the operator $H_{A}$ belongs to $C_{\text {red }}^{*}(\Gamma, \mathcal{K})$ for every $\zeta \in \rho\left(H_{A}\right)$.

Proof. Since $C_{\text {red }}^{*}(\Gamma, \mathcal{K})$ is closed in $\mathcal{L}(\mathcal{H})$ and $R_{A}(\zeta)$ is an analytic operator function on $\rho\left(H_{A}\right)$, it suffices to prove that $R_{A}(\zeta) \in C_{\text {red }}^{*}(\Gamma, \mathcal{K})$ when $\zeta$ runs over some semi-axis $\left(-\infty, \zeta_{0}\right)$. It is proved in [8] that $\exp \left(-t H^{0}\right) \in C_{\text {red }}^{*}(\Gamma, \mathcal{K})$ for all $t>0$; hence using the Laplace transform we get that $R^{0}(E) \in C_{\text {red }}^{*}(\Gamma, \mathcal{K})$ for every $E<0$. Put $V(E):=$ $R^{0}(E)-R_{A}(E)$; it remains to show that $V(E) \in C_{\text {red }}^{*}(\Gamma, \mathcal{K})$ for all $E$ in some interval $\left(-\infty, \zeta_{0}\right)$. We abbreviate

$$
M(\lambda, \mu ; \zeta):=[Q(\zeta)+A]_{\lambda \mu}^{-1} .
$$

According to Theorems 1 and 2 we can find constants $c_{E}<0$ and $c_{0}>0$ such that for all $E<c_{E}$

$$
|M(\lambda, \mu ; E)| \leq c_{0} \exp \left(-\tilde{c}_{0} d(\lambda, \mu)\right)
$$

and for every $f \in L^{2}(X)$ and $\zeta \in \rho\left(H_{A}\right)$

$$
V(\zeta) f=\sum_{\lambda \in \Lambda}\left(\sum_{\mu \in \Lambda} M(\lambda, \mu ; \zeta)\left\langle g_{\mu}(\bar{\zeta}) \mid f\right\rangle\right) g_{\lambda}(\zeta)
$$

Further, by Lemma 1 we can suppose that the following assertion is true: For any compact set $C \subset X$, any point $\kappa \in \mathrm{K}$, and any $E<c_{E}$ there is a constant $k(C, \kappa)$ such that

$$
\left[\int_{C}\left|g_{\lambda}(E)(x)\right|^{2} d x\right]^{1 / 2} \leq k \exp \left(-\tilde{c}_{0} d(\lambda, \kappa)\right) .
$$

Then we can choose $\tilde{c}_{0}$ in such a way that $\tilde{c}_{0}>3 \tilde{c}_{\Lambda}$ where $\tilde{c}_{\Lambda}$ is the constant from the proof of Lemma 2.

To see that $V(\zeta)$ is in $C_{r e d}^{*}(\Gamma, \mathcal{K})$, for $\operatorname{Re} \zeta<\zeta_{0}$, it is enough to show that

$$
\widehat{V(\zeta)}(\gamma) \in \mathcal{K}, \quad \gamma \in \Gamma
$$


and

$$
\sum_{\gamma \in \Gamma}\|\widehat{V(\zeta)}(\gamma)\|<\infty
$$

by Lemma F. We compute with (15) and (17)

$$
\widehat{V(\zeta)}(\gamma)=\sum_{\lambda, \mu \in \Lambda} M(\lambda, \mu ; \zeta)<g_{\mu}(\bar{\zeta}), \Phi^{-1}\left(\delta_{1}^{v}\right)>r_{F}\left(g_{\lambda}(\zeta) \circ \gamma^{-1}\right)
$$

Using the explicit form of $\Phi^{-1}$ we obtain

$$
\begin{aligned}
\left|<g_{\mu}(\bar{\zeta}), \Phi^{-1}\left(\delta_{1}^{v}\right)>\right| & =\left|<g_{\mu}(\bar{\zeta}), e_{F}(v)>\right| \\
& =\left|\int_{F} G^{0}(x, \mu ; \bar{\zeta}) \overline{v(x)} d x\right| \\
& \leq k \exp \left(-\tilde{c_{0}} d\left(\mu, k_{0}\right)\right)\|v\|_{L^{2}(F)}
\end{aligned}
$$

Moreover, from (18) it follows that

$$
\begin{aligned}
\left\|r_{\mathcal{F}}\left(g_{\lambda}(\zeta) \circ \gamma^{-1}\right)\right\|_{L^{2}(F)} & =\left[\int_{F}\left|G^{0}\left(\gamma^{-1} x, \lambda ; \zeta\right)\right|^{2} d x\right]^{1 / 2} \\
& \leq K \exp \left(-\tilde{c_{0}} d\left(\gamma \lambda, k_{0}\right)\right) .
\end{aligned}
$$

We observe next that

$$
d(\lambda, \mu)+d\left(\mu, \kappa_{0}\right)+d\left(\gamma \lambda, \kappa_{0}\right) \geq \frac{1}{3}\left(d\left(\lambda, \kappa_{0}\right)+d\left(\mu, \kappa_{0}\right)+d\left(\gamma^{-1} \kappa_{0}, \kappa_{0}\right)\right) .
$$

Since $\tilde{c_{0}}>3 \tilde{c_{\Lambda}}$, it follows from $(21 \mathrm{a}, \mathrm{b}),(16 \mathrm{~b}),(22)$, and Lemma $\mathrm{E}$ that the sum in (20) is norm convergent, uniformly in $\operatorname{Re} \zeta<\zeta_{0}$. Since all summands are operators of rank 1 we obtain (19a); in view of (22) we can sum over $\gamma \in \Gamma$ to verify (19b), by another application of Lemma $\mathrm{E}$. The proof is complete.

Corollary 1. Let $E_{1}, E_{2} \in \mathbf{R} \backslash \operatorname{spec} H_{A}$, and $E_{1} \leq E_{2}$. Then the spectral projector $P_{\left[E_{1}, E_{2}\right]}$ of $H_{A}$ belongs to $C_{\text {red }}^{*}(\Gamma, \mathcal{K})$.

Proof. Indeed, there exists a function $\varphi$ in $C_{0}^{\infty}(\mathbf{R})$ such that $P_{\left[E_{1}, E_{2}\right]}=\varphi\left(R_{A}(E)\right)$ for some $E<E_{1}$.

Fix now a number $E^{\prime} \in \mathbf{R}$ such that $E^{\prime}<\inf \operatorname{spec} H_{A}$ and consider the function

$$
N(E)= \begin{cases}\operatorname{Tr}_{\Gamma} P_{\left[E^{\prime}, E\right]}, & E \geq E^{\prime} \\ 0, & E<E^{\prime}\end{cases}
$$

It is clear that this function is independent of the choice of $E^{\prime}$. Moreover, $N(E)$ is constant on each gap of the spectrum of $H_{A}$ such that the values of $N(E)$ label in a natural way the gaps of $H_{A}[26]$.

Corollary 2 (Gap Labelling Theorem). The values of $N(E)$ on gaps of the spectrum of $H_{A}$ form a countable set of real numbers $\operatorname{Tr}^{*}\left(K_{0} C_{\text {red }}^{*}(\Gamma)\right)$ (here $K_{0} \mathcal{B}$ denotes the $K_{0}$-group of a $C^{*}$-algebra $\left.\mathcal{B}\right)$. 
Recall that $\Gamma$ is said to have the Kadison property if there exists a constant $c_{K}>0$ such that $\operatorname{Tr}_{\Gamma} P \geq c_{K}$ for every non-zero orthogonal projector from $C_{\text {red }}^{*}(\Gamma, \mathcal{K})$.

Corollary 3. If $\Gamma$ has the Kadison property, then the spectrum of $H_{A}$ has band structure.

\section{Acknowledgements}

The second named author gratefully acknowledges a grant of Volkswagen-Stiftung. He is also very grateful to the RFFI Foundation (Grant No 96-01-00074) for financial support, as well as to Humboldt University at Berlin for its warm hospitality.

\section{References}

1. B. S. Pavlov. The theory of extensions and explicitly solvable models (in Russian). Uspekhi Mat. Nauk 42, No 6 (1987), 99-131; Engl. transl.: Russ. Math. Surv. 42, No 6 (1987), 127-168.

2. S. Albeverio, F. Gesztesy, R. Høegh-Krohn, and H. Holden. Solvable models in quantum mechanics. Springer-Verlag, Berlin etc., 1988.

3. R. Kronig and W. G. Penney. Quantum mechanics of electrons in crystal lattices. Proc. Roy. Soc. (London) 130A (1931), 499-513.

4. A. Grossmann, R. Høegh-Krohn, and M. Mebkhout. The one-particle theory of periodic point interactions. Comm. Math. Phys. 77 (1080), 87-100.

5. Yu. E. Karpeshina. Spectrum and eigenfunctions of Schrödinger operator with zerorange potential of the homogenous lattice type in three dimensional space (in Russian). Teor. i. Mat. Fiz. 57 (1983), 304-313; Engl. transl.: Theor. and Math. Phys., 57 (1983), 1156-1162.

6. S. Albeverio, F. Gesztesy, R. Høegh-Krohn, and H. Holden. Point interactions in two dimensions: Basic properties, approximations and applications to solid state physics. J. reine u. angew. Math. 380 (1987), 87-107.

7. T. Sunada. Group $C^{*}$-algebras and the spectrum of a periodic Schrödinger operator on a manifold. Can. J. Math. 44 (1992), 180-193.

8. J. Brüning and T. Sunada. On the spectrum of periodic elliptic operators. Nagoya Math. J. 126 (1992), 159-171.

9. J. Brüning and T. Sunada. On the spectrum of gauge-periodic elliptic operators. Astérisque 210 (1992), 65-74.

10. M. G. Krein and H. K. Langer. Defect subspace and generalized resolvents of an Hermitian operators in the space $\Pi_{\kappa}$ (in Russian). Funk. Anal. i Prilozhen. 5, No 2 (1971), 59-71; Engl. transl.: Funct. Anal. and its Appl. 5 (1971), 217-228.

11. J. Brüning and V. A. Geyler. On the spectrum of gauge periodic point perturbations on the Lobachevsky plane. Preprint 341 SFB 288, Berlin, 1998.

12. T. Sunada. Euclidean versus non-euclidean aspects in spectral geometry. Progr. Theor. Phys. Suppl. 116 (1994), 235-250. 
13. N. E. Hurt. Quantum chaos and mesoscopic systems. Mathematical methods in the quantum signatures of chaos. Kluwer Ac. Publ., Dordrecht etc. , 1997.

14. M. L. Leadbeater, C. L. Foden, J. H. Burrougher, e.a. Magnetotransport in a nonplanar two-dimensional electron gas. Phys. Rev. B. 52 (1995), 8629-8632.

15. D. B. Efremov and M. A. Shubin. Spectral asymptotics of elliptic operators of the Schrödinger type on the Lobachevsky space (in Russian). Trudy Sem. I. G. Petrovsky 15 (1991), 3-32.

16. M. A. Shubin. Spectral theory of elliptic operators on non-compact manifolds. Astérisque 207 (1992), 35-108.

17. A. V. Bukhvalov. Applicatons of the method of the theory of order bounded operators in the $L^{p}$-spaces (in Russian). Uspekhi Mat. Nauk 38, No 6 (1983), 37-83.

18. V. B. Korotkov. Integral operators (in Russian). Nauka, Novosibirsk (1983).

19. V. A. Geyler, V. A. Margulis, and I. I. Chuchaev. Zero-range potentials and Carleman operators (in Russian). Sibir. Mat. Zhurn. 36 (1995), 828-841; Engl. transl.: Siberian Math. J. 36 (1995), 714-726.

20. P. R. Halmos and V. S. Sunder. Bounded linear operators on $L^{2}$-spaces.SpringerVerlag, New York etc., 1978.

21. M. A. Shubin. Pseudo-difference operators and their Green functions (in Russian). Izv. AN SSSR. Ser. Mat. 49 (1985), 652-671; Engl. transl.: Math. USSR. Izvestiya. 26 (1986), 605-622.

22. S. A. Gredeskul, M. Zusman, Y. Avishai, and M. Ya. Azbel. Spectral properties and localization of an electron in a two-dimensional system with point scatterers in a magnetic field. Phys. Reps. 288 (1997), 223-257.

23. V. A. Geyler. The two-dimensional Schrödinger operators with a uniform magnetic field and its perturbation by periodic zero-range potentials (in Russian). Algebra $i$ Analiz 3, No 3 (1991), 1-48; Engl. transl.: St.-Petersburg Math. J. 3 (1992), 489-532.

24. S. Albeverio and V. A. Geyler. The band structure of the general periodic Schrödinger operator with point interactions (to be published).

25. Yu. G. Shondin. Semibounded local Hamiltonians for perturbation of the Laplacian supported by curves with angle points in $\mathbf{R}^{4}$ (in Russian). Teoret. i Mat. Fiz. 106 (1996), 179-199.

26. J. Bellissard. Gap labelling theorems for Schrödinger operators. In: From Number Theory to Physics. Eds. Waldschmidt M. e.a. Springer-Verlag, Berlin etc., 1992. P. 538-630.

J. Brüning, Institute of Mathematics, Humboldt University at Berlin, Unter den Linden 6, 10099 Berlin, Germany; e-mail: bruening@mathematik.hu-berlin.de

V.A.Geyler, Department of Mathematics, Mordovian State University, 430000 Saransk, Russia; e-mail: root@mathan.mordovia.su 